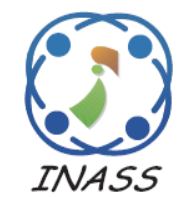

\title{
Genetic algorithm based Backup Route Establishment for QoS Routing
}

\author{
Rama RaoAdimalla $^{1 *}$ Vatsavayi Valli Kumari $^{2} \quad$ Ch Satyananda Reddy ${ }^{2}$ \\ ${ }^{1}$ Lendi Institute of Engineering and Technology,Andhra Pradesh, India \\ ${ }^{2}$ Andhra University, India \\ * Corresponding author's Email: ramarao.a79@gmail.com
}

\begin{abstract}
Mobile ad-hoc network face a serious issue of frequent change of network topology which leads to the stability and reliability problems of routing. Many routing schemes such as multi-path routing and backup path routing are developed to increase the link reliability in the literature. Accordingly, we propose to design a backup route establishment for QoS routing protocol. In this protocol, the potential failures of network and node are identified and a backup routing is initiated. For detection of failures, a path evaluation function is determined based on the metrics energy drain rate and interference, congestion status are measured. The primary path satisfying the QoS parameters node's static resource capacity, dynamic resource availability, neighborhood quality, link quality is established. Once failure is detected, back up routes are established and transmission is redirected on these back up routes. Simulation results show that the proposed protocol has reduced recovery delay and improved throughput as compared with the existing protocol. The improvement of $68 \%$ in terms of delay, $30 \%$ in terms of delivery ratio and $83 \%$ in terms of throughput are achieved for the proposed routing protocol.
\end{abstract}

Keywords: MANET, QoS, Routing, Genetic Algorithm (GA), Backup routing, Link quality.

\section{Introduction}

MANET is an autonomous collection of mobile systems that communicate over relatively bandwidth constrained wireless links. As the nodes are mobile, the network topology changes rapidly and randomly with time. The network is decentralized, where network activity such as discovering the topology and delivering messages are executed by the node themselves that means routing functionality has to be integrated in mobile nodes [1]. The Characteristics of MANET like high mobility and frequent link failures results in low throughput and high end-to-end delay. Due to high demand of MANETs for transferring multimedia applications such as voice, video and data, leads to the need to provide QoS support [2]. The characteristics of MANETs such as their dynamic topology coupled with the characteristic of the wireless communication medium make Quality of Service condition a complicated challenge [3].
The main aim of QoS is to provide preferential delivery service for the application that need it by ensuring sufficient bandwidth, controlling latency and jitter, and even reducing data loss. The issue how to establish a connection with QoS requirement across multiple domains operated by different operator is an important and challenging research [4]. In traditional routing, the main aim of routing algorithm is to find the least-coast path from sender to receiver, Whereas QoS routing has two objectives. The first objective is to find the path that meet with QoS requirements called as feasible paths. The second objective is to optimize the global network resource utilization so that network can contain as many QoS request as possible [5]. These two objectives should be effectively included in the optimization algorithm function to find the bet suitable routing oath for data transmission in MANET.

In recent days, the major issues to provide QoS in MANET is due to the common characteristics of wireless ad hoc networks like high node mobility, 
distributed channel access and weak radio signal effect. This entire factor can be overcome with the cooperation of different protocol layers to provide QoS [6, 7]. When delivering the QoS to frequent change of network topology, backup routing is important. The backup path routing is another type of multiple path routings. Multiple short backup routes are attached to the active primary route $[8,9]$. Data packets are delivered along the primary route rather than distributed them among the backup routes. In general, mobile nodes in the primary route should exchange the routing information with their neighbor nodes [9, 10]. Therefore, the scope of backup path is limited to the vicinity and the length of back path is also restricted. When the primary route is disconnected (due to the absence of relay nodes or radio shadowing), the data packets which are on transmission can be salvaged by re-directing them into the backup route without any delay [11].

In this paper, backup route establishment technique for QoS routing protocol is proposed. The primary path is selected using genetic algorithm by modifying the fitness function incorporating node's static resource capacity, dynamic resource availability, neighborhood quality, link quality. Then, the backup path is established using the path evaluation function which considers three important parameters like, energy, interference and congestion. Every intermediate node which comes under primary path is validated using path evaluation function and the second path is established if any nodes are depleted based on the path evaluation function.

The contribution of this paper is in the primary path selection where, GA is utilized to find the possible number of routing paths based on the link metrics. At first, population is initialized and fitness value is calculated for every chromosome. The fitness function of each route in the network is based on various parameters like, node's static resource capacity, dynamic resource availability, neighborhood quality, link quality in such a way that it satisfies the set of QoS requirement. Then, cross over operator is applied for several times to create $\mathrm{m}$ new children for the new population and mutation operator is applied on the chromosomes in the mating pool. This process is repeated for the required number of iterations and the chromosome having the best fitness is selected as the final route in the primary path selection. Once the primary path is established, data communication can happen based on the selected routes. Then, the route failure is checked by the path evaluation function to establish the secondary route which is selected as a backup path for the data session. The main advantage of the proposed protocol is that it has reduced the recovery delay and improved throughput.

The paper is organized as follows: Section 2 presents the review of related works and section 3 presents the proposed Backup Route Establishment for QoS Routing Protocol in MANET. Section 4 discusses the simulation Results and section presents the conclusion of the paper.

\section{Review of related works}

Literature presents different works for multipath routing protocol by considering QoS parameters. Accordingly, Rango and Tropea [12] have proposed a routing algorithm, called Load Balancing and Energy aware ARAMA (LBE-ARAMA). This algorithm was based on Swarm Intelligence approach. It discovered minimum multipath disjoint routing (MDR) for load balancing and energy conservation. The Round Trip Time (RTT) delay is evaluated along the path from source to destination. The algorithm was able to satisfy multiple metrics for a multi-objective optimization like end-to-end delay, load balancing and energy saving. Canales et al. [13] have proposed a distributed adaptive admission control procedure based on a cross-layer QoS Routing for estimating end-to-end available bandwidth. They have developed an effective TDMA MAC protocol which allows to carryout prompt resource reservation for point-to-point communications. Then, a distributed admission control based on the joint operation of a modified AODV with the TDMA MAC structure was proposed. It guarantees an end-to-end QoS provision with an appropriate adaptation to the variability of MANETs. The disadvantage is it does not provide the guaranteed bandwidth for new resources.

Ya-li et al. [14] have proposed an improved ant colony-based multi-constrained QoS energy-saving routing algorithm (IAMQER). The proposed algorithm improves the performance of network in multi-constrained QoS routing based on the local node information such as node queue length, number of data packets forwarded and node residual energy.To reduce average energy consumption, the algorithm adjusts nodes transmit power dynamically according to the different distance between two nodes. This method does not work well for the high network load and more complete network topology.

Llewellyn et al. [15] have presented a distributed fault-tolerant routing protocol for QoS support in mobile ad hoc networks, which mitigates disruption time under network failures. The work demonstrates that the traditional method of rerouting QoS traffic 
from the source given a link failure yields serious negative QoS disruption consequences; an efficient local fault-tolerant algorithm can significantly mitigate the time required to re-establish a connection. Lowering the reconnection time reduces QoS disruptions. The dropped packet and throughput results reflected similar outcomes. EFDCB protocol used in this paper does not perform updates fast enough to have an accurate view of the network state.

Hanzo et al. [16] have proposed and evaluated new solutions for improving the performance of QAR and AC protocols in the face of mobility, shadowing, and varying link SINR. It is found that proactively maintaining backup routes for active sessions, adapting transmission rates, and routing around temporarily low-SINR links can noticeably improve the reliability of assured throughput services.This method affect the ability of the protocol to maintain the desired throughput of already admitted sessions as well. Gu et al. [17] have proposed a hierarchical architecture of the return channel in ICBN and an NAK-based hierarchical ARQ scheme (H-ARQ). The H-ARQ scheme can alleviate the NAK implosion and balance the retransmission traffic between broadcast and communication networks. The users are divided into regions in the communication network, and each region has a Local Recovery Router (LRR), which cooperates with the Data Broadcast Server (DBS) to decide the retransmission method. The impact of the ARQ scheme on the data broadcast. In this method, the efficiency of the broadcast downlink fall sharply because there are too many retransmitted packets.

Recently, backup routing protocols are developed to handle the frequent change in network topology. Accordingly, Wen-Tsuen Chen and Lee [11] have developed the Dynamic AODV Backup Routing Protocol (DABR) to enhance the Ad hoc on-Demand Distance Vector (AODV) routing in dense mobile ad-hoc networks. The DABR follows the route discovery mechanism of AODV and dynamically calculates the backup routes. Upon the failure of primary route, data packets can be salvaged by redirecting them to the backup routes. The simulation results showed that the link reliability of DABR is higher than the conventional AODV while the overhead was controlled. Chaudhary et al. [18] have proposed a scheme to improve existing on-demand routing protocols by creating a mesh and providing multiple alternate routes with the assurance of no link failure. Their algorithm established the link failure prediction embedded within the existing protocol.This scheme does not perform well under heavy traffic networks and frequently breaks in primary as well as alternate routes due to high mobility of nodes.

In [19], two schemes such as, AODV-ABR and AODV-ABL were developed to increase the adaptation of routing protocols to topology changes by modifying AODV-BR. One of the important issues for the modern networks is the Quality of Service (QoS). This method does not consider the QoS issues. Jaisankar and Saravanan [20] have presented a multipath routing scheme to provide better performance and scalability by computing multiple routes in a single route discovery. Also, it reduced the routing overhead by using secondary paths. This scheme computes combination of the node-disjoint path and fail-safe paths for multiple routes and provides all the intermediate nodes of the primary path with multiple routes to destination. The disadvantage is that the multipath routing scheme is not secure. Zamani and Soltanaghaei [21] have proposed a method, called M-AODV, which is a type of overhearing backup protocol, based on AODV, is presented. The simulation results of this protocol, applied by NS2 simulator, showed the improvement of packet delivery rate and reduction of overhead and delay. This method was secure against only for wormhole and black hole attacks. Also, the two parameters, power consumption and electromagnetic interference can affect the results of the simulation.

\section{Proposed backup route establishment for QoS routing protocol in MANET}

This section presents the block diagram of the proposed backup route establishment for QoS routing protocol. The proposed mechanism contains two important steps such as, i) primary route establishment, ii) backup route establishment. In the first phase, GA is applied for discovering the routes. In the second phase, backup routing protocol is established. Here the probable breakdown of network and node are identified and a backup routing is initiated. Therefore for the detection of failures, a path evaluation function is determined [14] based on the metrics energy drain rate [12] and interference [13], congestion status, are measured. Once failure is detected, back up routes are established similar to our first paper and transmission is redirected on these back up routes. Fig. 1 shows the block diagram of the proposed Backup Route Establishment for QoS Routing Protocol. 


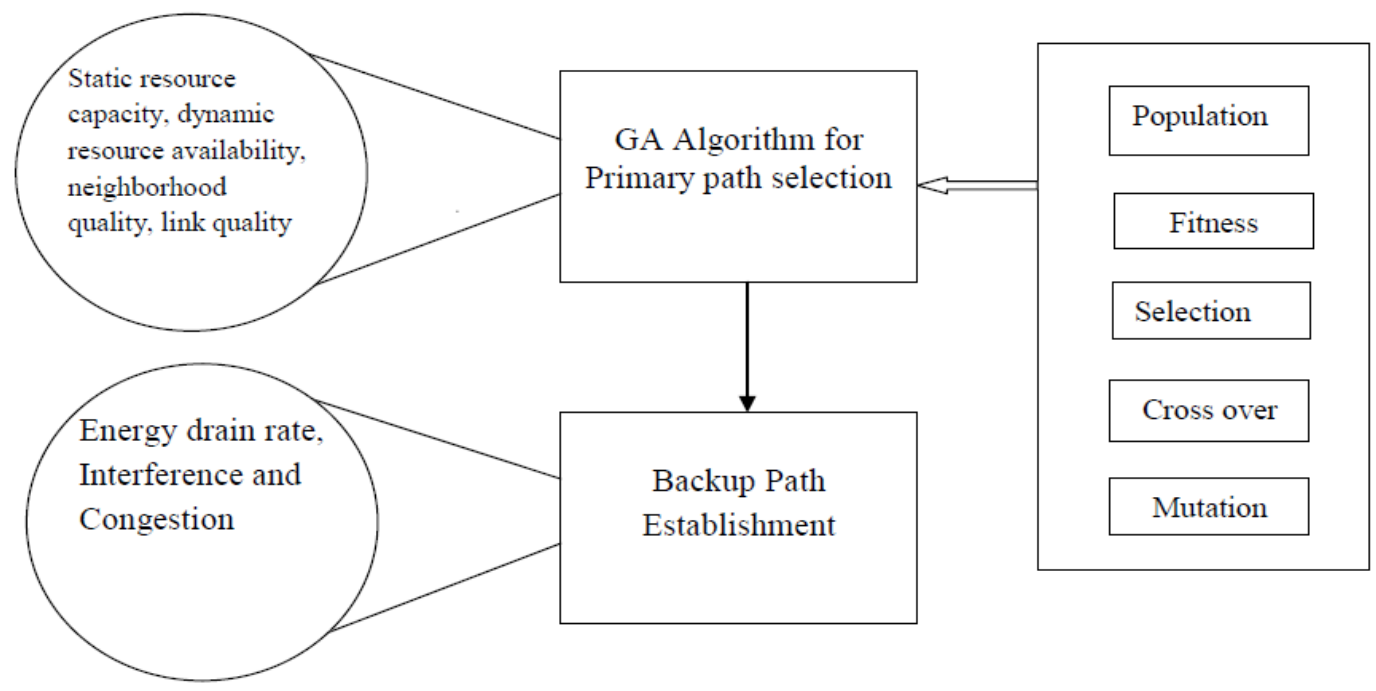

Figure.1Block diagram of the proposed Backup Route Establishment for QoS Routing Protocol

\subsection{Primary path selection by GA-based routing protocol}

A communication network is simulated as a directed graph $G(V, E)$, where $V$ is the set of nodes representing the routers and $E$ is the set of edges representing the links that connect between the routers. For a network supporting multiple QoS metrics, each edge $(i, j)$ is associated with 4 independent metrics of having numerical value from 0 to 1 . The values of static resource capacity, dynamic resource availability, neighborhood quality, link quality are also initialized.

GA algorithm for route discovery: In this primary path selection algorithm, GA is utilized to find the possible number of routing paths based on the link metrics.

Initialization: At first, population is initialized. The values of static resource capacity, dynamic resource availability, neighborhood quality, link quality. Each chromosome value is calculated. Mating pool is created and which consists of all the chromosomes in the current population.

Fitness function: The fitness function of each route in the network is based on various parameters like node's static resource capacity, dynamic resource availability, neighborhood quality, link quality in such a way that it satisfies set of QoS requirement. Each parameter is assigned with a weight. These weighted parameters are combined into a single function, which is known as fitness function. The fitness function must minimize the total cost TC and it is calculated using the following equation

$$
T C=\alpha \times S R C+\beta \times D R A+\lambda \times L Q+\delta \times N Q
$$

Where, SRC is the static resource capacity, DRA is dynamic resource availability, NQ is neighborhood quality and LQ is link quality. $\alpha, \beta, \lambda$ and $\delta$ are the weight values of SRC, DRA, LQ and NQ respectively.

Cross over: Cross over operator function is applied for several times to create $\mathrm{m}$ new children for the new population. The parents are selected using the selection operator. Each pair of parent chromosome can only mate once and the children produced are only accepted into the new population if they are not similar to the previously produced children. This is done to diversify the search and discourage convergence. If all the possible pair of parents have mated and there are still not enough children to populate the new population, select members from the previous population using the selection operator to fill in the new population.

Mutation: Apply mutation operator is applied on the chromosomes in the mating pool. Each chromosome has a certain probability to be mutated. Mutation result must not be the same as any of the chromosome in the current population or else it would be ignored. Replace the worst (the one with the lowest fitness value) chromosome produced by the genetic operators (crossover and mutation) with the best chromosome in the previous population.

Termination: This process is repeated for the required number of iterations and the chromosome having the best fitness is selected as the final route in the primary path selection. By implementing this method, we are minimizing QoS values on links from source to destination and increasing the possibility for the path to satisfy the given QoS requirement and hence we can overcome the MCP problem. The algorithm for primary path selection is given in Table 1. 
Table 1. Algorithm (1): GA-Based Routing Algorithm

\begin{tabular}{|l|l|}
\hline 1 & Algorithm-1: Primary Path Selection \\
\hline 2 & Parameters \\
\hline 3 & SRC = Static Resource Capacity \\
\hline 4 & DRA = Dynamic Resource Availability \\
\hline 5 & NQ = Neighborhood Quality \\
\hline 6 & LQ = Link Quality \\
\hline 7 & M = Population Size \\
\hline 8 & TV = Threshold Value \\
\hline 9 & Start \\
\hline 10 & Randomly initialize the initial population \\
\hline 11 & Calculate the SRC, DRA, NQ, LQ \\
\hline 12 & $\begin{array}{l}\text { Calculate fitness function for each chromosome } \\
\text { using the equation (8) }\end{array}$ \\
\hline 13 & $\begin{array}{c}\text { Create the mating pool, which consists of all the } \\
\text { chromosomes in the current population }\end{array}$ \\
\hline 14 & $\begin{array}{c}\text { Apply the crossover operator several times to } \\
\text { create m new children for the new population }\end{array}$ \\
\hline 15 & If (fitness value < TV) \\
\hline 16 & $\begin{array}{c}\text { Replace the worst value with best value using } \\
\text { the crossover operation }\end{array}$ \\
\hline 17 & Repeat maximum paths found \\
\hline 18 & ? \\
\hline 19 & \\
\hline 20 & End \\
\hline
\end{tabular}

Table 2. Algorithm (2): Backup Routing establishment Algorithm

\begin{tabular}{|l|l|}
\hline 1 & Algorithm-2: Backup Path Establishment \\
\hline 2 & Parameters: \\
\hline 3 & $\mathrm{~S}-$ Source, \\
\hline 4 & $D$-Destination, \\
\hline 5 & $N_{i}, \mathrm{i}=1,2 \ldots \mathrm{k}-$ Intermediate nodes, \\
\hline 6 & $P E$ - path evaluation function, \\
\hline 7 & $P E T h-$ threshold value for path evaluation function, \\
\hline 8 & $\quad R F N$ - Route failure notification \\
\hline 9 & Start \\
\hline 10 & $\begin{array}{l}\text { Establish the primary path between the S and D } \\
\text { using Algorithm-1 }\end{array}$ \\
\hline 11 & $\begin{array}{l}\text { S transmits the data towards the destination } \\
\text { through the established primary path. }\end{array}$ \\
\hline 12 & Each Ni along the primary path estimates PE \\
\hline 13 & If PE(Ni) $>$ PETh, then \\
\hline 14 & Ni transmit a path failure notification to S \\
\hline 15 & $\begin{array}{l}\text { S selects the next best path established } \\
\text { through Algorithm-1 as backup path }\end{array}$ \\
\hline 16 & $\begin{array}{l}\text { S resumes the transmission to D through the } \\
\text { backup path }\end{array}$ \\
\hline 17 & End if \\
\hline 18 & End \\
\hline
\end{tabular}

\subsection{Backup path selection by path evaluation function}

Once the primary path is established, data communication can happen based on the selected routes. Then, the route failure is checked by the path evaluation function to establish the secondary route which is selected as a backup path for the data session. Path evaluation function (PE) is a metric which presents the performance of route from source node to destination node. It is related to the metrics energy drain rate, interference and congestion status. The mathematical model used to find the path evaluation function $P E_{P^{k}}$ is given by,

$$
\mathrm{PE}_{\mathrm{k}}=\omega_{1} \cdot E D r_{k}+\omega_{2} \cdot \operatorname{Int}_{k}+\omega_{3} \cdot C_{k}
$$

Where, EDr, Int and $\mathrm{C}$ are energy drain rate, interference and congestion status estimated. $\omega_{1}, \omega$ 1 and $\omega_{3}$ are the positive weight values of EDr, Int and $\mathrm{C}$, respectively. They are in the range between 0 to 1.According to (2), path evaluation function is directly proportional to EDr, Int and C. (ie) If energy drain rate, interference and congestion status are high, $\mathrm{PE}$ is high, which indicates the possibility of failure of the path.

Energy Drain rate:Let us consider that each node $i$ monitors its energy consumption caused by the transmission, reception and overhearing activities and computes the energy drain rate, denoted by $E D r_{i}(t)$. This is made for every $\Delta \mathrm{t}$ seconds sampling interval by averaging the amount of energy consumption and estimating the energy dissipation per second during the past $\Delta \mathrm{t}$ seconds. The actual value of $E D r_{i}(t)$ is calculated by utilizing the well-known exponential weighted moving average method applied to the drain rate values $E D r_{i}(t-1)$ and $E D_{\text {curr }, i}(t)$,

$$
E D r_{i}(t)=\delta \cdot E D r_{i}(t-\Delta t)+(1-\delta) \cdot E D_{\text {curr }, i}(t)
$$

Where $\operatorname{EDr}_{i}(t)$ is Energy drain rate, $\Delta \mathrm{t}$ is the previous time instant. $\delta$ is the positive weight. The current EDri (t) is calculated as follows:

$$
E D r_{\text {curr }, i}(t)=\frac{E_{i}(t)-E_{i}(t-\Delta t)}{\Delta t}
$$

Where $\Delta \mathrm{t}$ is the previous time instant.

Interference metric:The interference metric is used for the detection of failures. A reliable link requires not only a Signal to Interference Ratio received level $\left(\operatorname{SIR}_{\mathrm{rx}}\right)$ high enough to correctly decode the information (over SIRth), but also a minimum useful signal power $\mathrm{P}_{\mathrm{rx}, \min }$. It is given by,

$$
\text { Int }=P_{r x, \text { min }}: S N R=\frac{P_{r x, \text { min }}}{P_{\text {noise }}}=S I R_{t h}+\Delta S I R(5)
$$

Where, SNR is Signal to noise ratio, $P_{r x, \text { min }}$ is Minimum received power, SIR is Signal to Interference Ratio. $\mathrm{P}_{\text {noise }}$ is the thermal noise.

Congestion Status:The $\mathrm{C}_{\mathrm{i}}$ metric is used to describe congestion status of a node $i$. It is proportional to node queue length, the number of forwarded data packets and the residual energy of node.

$$
C_{i}(t)=a \cdot Q_{i}^{\text {unit }}(t)+b \cdot T_{i}^{\text {unit }}(t)
$$

Where, $\mathrm{a}$ and $\mathrm{b}$ are positive weights, $Q_{i}^{\text {unit }}(t)$ is node unit queue length at current time step, $T_{i}^{\text {unit }}(t)$ 
is node unit forwarding packet number at current time step. $\mathrm{C}_{\mathrm{i}}(\mathrm{t})$ is the congestion status of node $\mathrm{i}$. Table 2 shows the algorithmic description of Backup Routing establishment Algorithm.

\section{Results and discussion}

This section presents the simulation of MANET and the experimental results of the proposed backup routing protocol. Here, comparative analysis based on three metrics is also performed.

\subsection{Simulation parameters}

We use NS2 [22] to simulate our proposed Backup Route Establishment for QoS Routing Protocol (BREQRP). We use the IEEE 802.11 for MANETs as the MAC layer protocol. It has the functionality to notify the network layer about link breakage. In our simulation, the packet sending rate is varied as $250,500,750$ and $1000 \mathrm{~Kb}$. The area size is 1250 meter x 1250 meter square region for 50 seconds simulation time. The simulated traffic is Constant Bit Rate (CBR). Our simulation settings and parameters are summarized in Table 3.

\subsection{Performance metrics}

We evaluate performance of the new protocol mainly according to the following parameters. We compare the IAMQER protocol with our proposed BREQRP protocol. Average Packet Delivery Ratio: It is the ratio of the number of packets received successfully and the total number of packets transmitted. Average end-to-end delay: The end-toend-delay is averaged over all surviving data packets from the sources to the destinations. Throughput: The throughput is the amount of data that can be sent from the sources to the destination.

\subsection{Comparative analysis}

This section presents the comparative analysis of the proposed back up routing protocol using different data rate, number of nodes and speed.

i) Based on Rate: In our first experiment, we are varying the data rate from 250 to $1000 \mathrm{~Kb}$. Fig. 2 to 4 shows the results of delay, delivery ratio and throughput by varying the rate from $250 \mathrm{~Kb}$ to $1000 \mathrm{~Kb}$ for the CBR traffic in BREQRP and IAMQER protocols. From Fig. 2, we understand that the proposed BREQRP achieved the DelRatio of 1005 when the data rate is $250 \mathrm{~Kb}$. Fig. 3 shows the delay values of various data rate. From this figure, we understand that the proposed BREQRP achieved the delay of zero for the data arte of $250 \mathrm{~Kb}$.
Also, we note that the throughput of the proposed BREQRP is increase when the data rate is increased. When comparing the performance of the two protocols, we infer that BREQRP outperforms IAMQER by $85 \%$ in terms of delay, $79 \%$ in terms of delivery ratio and $72 \%$ in terms of throughput.

Table 3. Simulation parameters

\begin{tabular}{|l|l|}
\hline No. of Nodes & $30,50,70,90$ and 110 \\
\hline Area & $1250 \times 1250$ \\
\hline MAC & 802.11 \\
\hline Simulation Time & $50 \mathrm{sec}$ \\
\hline Traffic Source & CBR \\
\hline Rate & $250,500,750$ and $1000 \mathrm{~Kb}$ \\
\hline Propagation & TwoRayGround \\
\hline Antenna & OmniAntenna \\
\hline Speed & $10,20,30,40$ and $50 \mathrm{~m} / \mathrm{s}$ \\
\hline
\end{tabular}

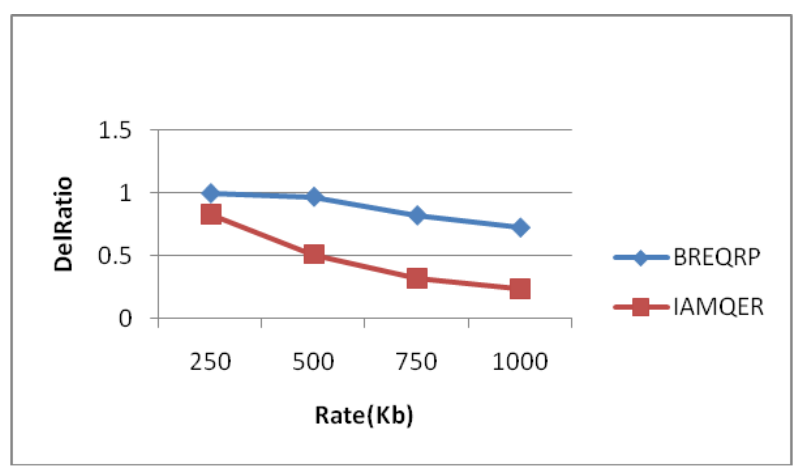

Figure. 2 Rate Vs Delivery Ratio

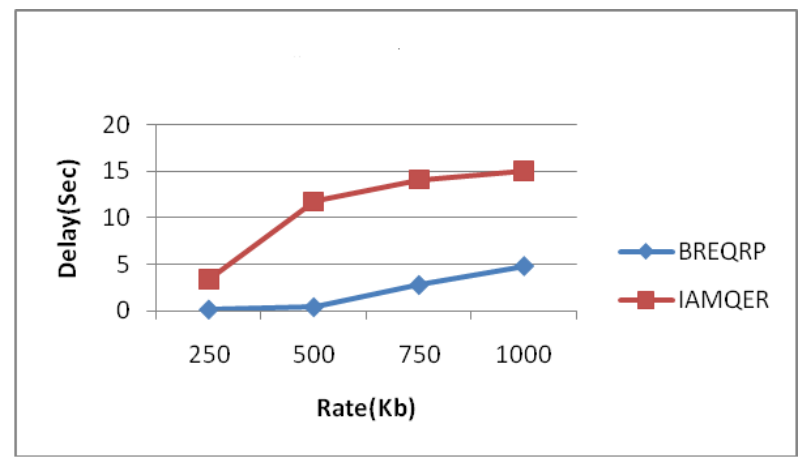

Figure. 3 Rate Vs delay

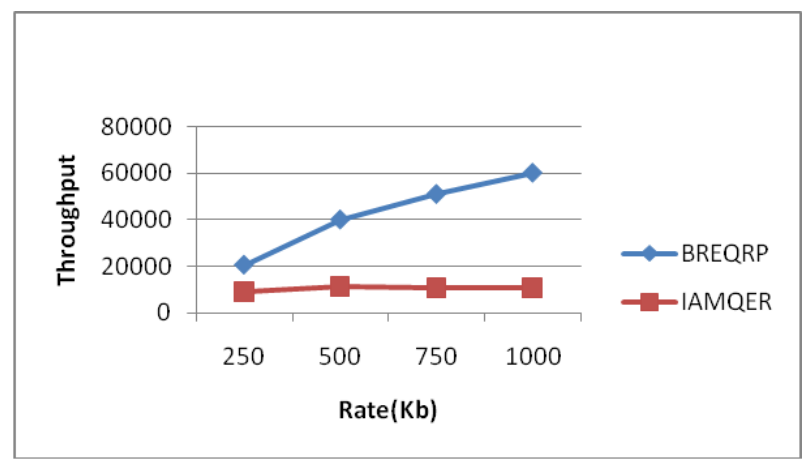

Figure. 4 Rate Vs Throughput 
ii) Based on Nodes: In our second experiment we are varying the number of nodes as 30,50,70,90 and 110 for CBR traffic. Fig. 5 to 7 show the results of delay, delivery ratio and throughput by varying the nodes from 30 to 110 for the CBR traffic in BREQRP and IAMQER protocols. From Fig. 5, we identified that the DelRatio of the proposed BREQRP is 0.4 for 110 nodes where, the existing reached only the value of 0.2. From Fig. 6, the delay value of the proposed BREQRP is $12 \mathrm{sec}$ which is less that $20 \mathrm{sec}$ achieved by the existing algorithm. Fig. 7 shows the improvement of the proposed BREQRP in terms of throughput. When comparing the performance of the two protocols, we infer that BREQRP outperforms IAMQER by 53\% in terms of delay, $47 \%$ in terms of delivery ratio and $75 \%$ in terms of throughput.

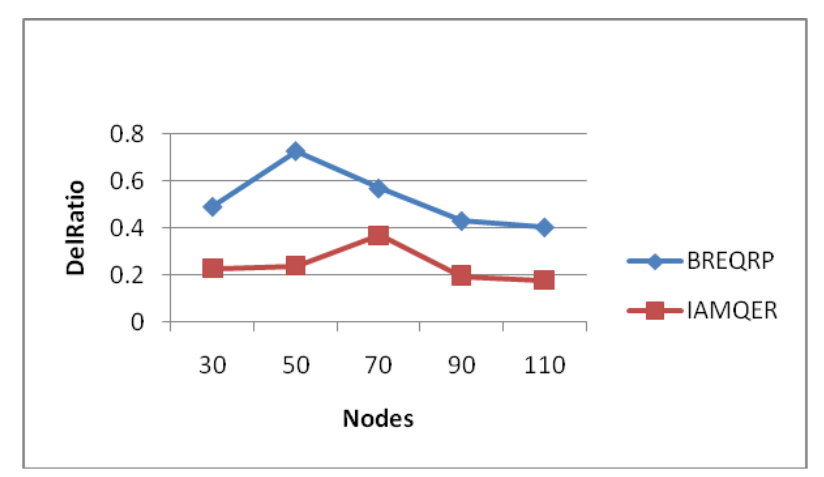

Figure. 5 Nodes Vs Delivery Ratio

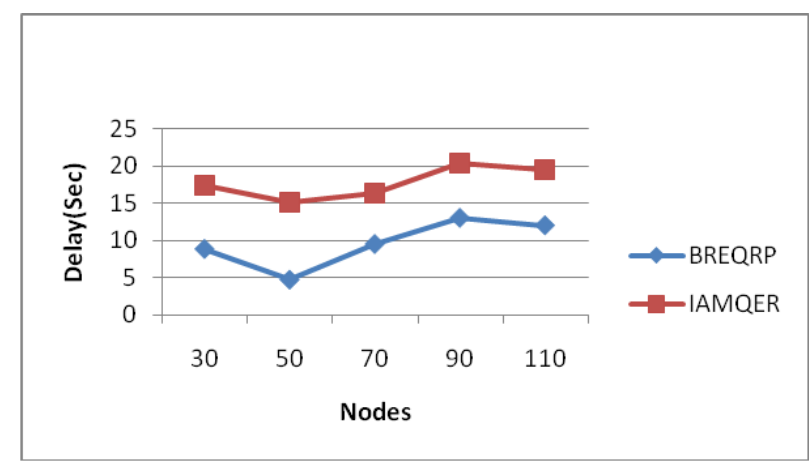

Figure. 6 Nodes Vs Delay

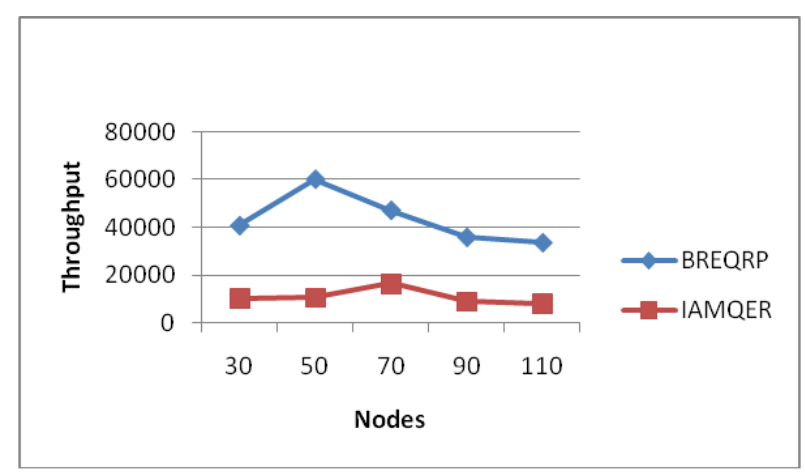

Figure. 7 Nodes Vs Throughput
Based on Speed: In our third experiment we are varying the mobile speed as $10,20,30$ and $40 \mathrm{~m} / \mathrm{s}$ for CBR traffic. Fig. 8 to 10 show the results of delay, delivery ratio and throughput by varying the speed from 10 to $40 \mathrm{~m} / \mathrm{s}$ for the CBR traffic in BREQRP and IAMQER protocols. When analyzing Fig. 8, the proposed BREQRP achieved the DelRatio of 0.75 which is higher than the existing protocol. Similarly, Fig. 9 shows that the proposed BREQRP obtained the delay of $4 \mathrm{sec}$ which is less that the existing algorithm for the speed of $10 \mathrm{~m} / \mathrm{sec}$. Fig. 10 implies that the proposed BREQRP When comparing the performance of the two protocols, we infer that throughput is decreased when the speed is increased for the proposed BREQRP. Overall, the proposed BREQRP outperforms IAMQER by $68 \%$ in terms of delay, $30 \%$ in terms of delivery ratio and $83 \%$ in terms of throughput.

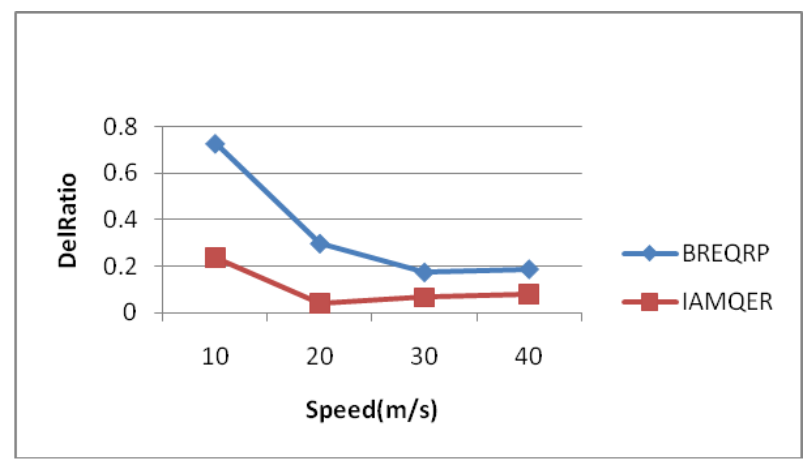

Figure. 8 Speed Vs Delivery Ratio

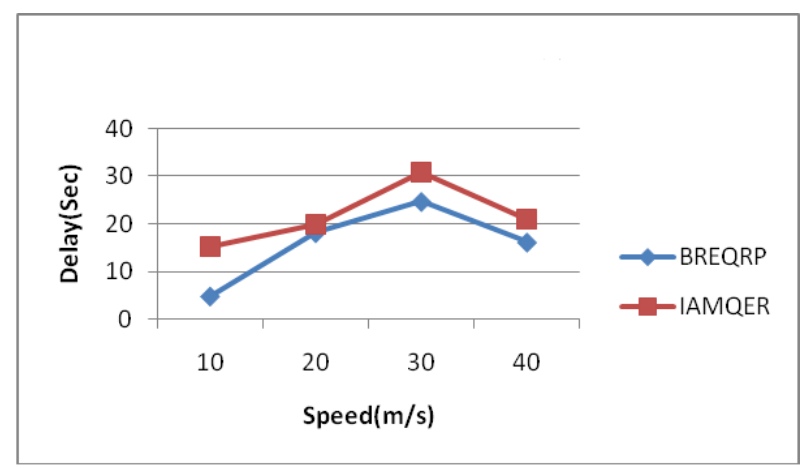

Figure. 9 Speed Vs Delay

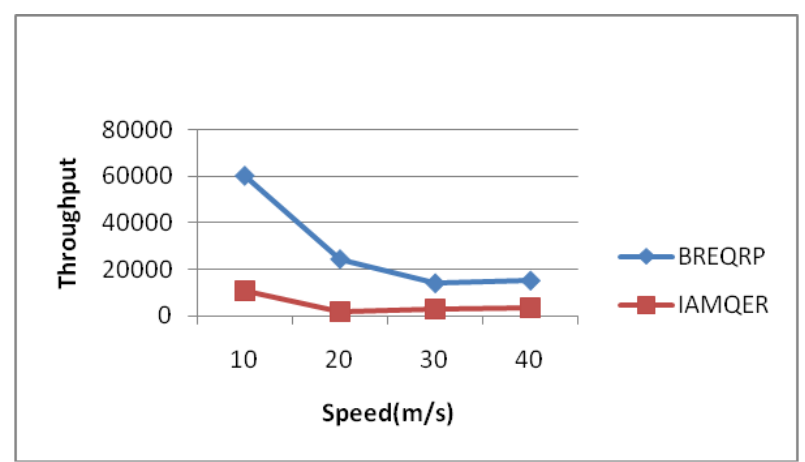

Figure. 10 Speed Vs Throughput 


\subsection{Discussion}

Table 4 shows the comparative discussion of the different protocols based on delay and delivery ratio for the fixed rate of $250 \mathrm{~Kb}$. For the data rate of 250 $\mathrm{kb}$, the delay is zero for the proposed BREQRP protocol and the delay for IAMQER protocol is 4 sec. For the same data rate, the protocols such as, AMRA and MAREERP achieved the delay of 7.5 sec and $6 \mathrm{sec}$ respectively. The other algorithms namely, Dijkstra, IAMQER, and NQoS-AODV achieved the delay rate of $8.8 \mathrm{sec}, 9.5 \mathrm{sec}$, and $9 \mathrm{sec}$ respectively for the same data rate. While compared to all the previous methods, the proposed method outperformed in terms of delay. On the other hand, the proposed BREQRP protocol achieved the delivery ratio of 1 for the data rate of $250 \mathrm{~kb}$ while the IAMQER protocol achieved the delivery ratio of 0.8 . The delivery ratio of the protocols such as, AMRA and MAREERP are 0.22 and 0.3 respectively, for the same data rate. The table 4 clearly mentioned that the delay and delivery ratio of the proposed method is better than the existing methods. The improved performance is achieved due to the behaviour of the genetic algorithm to converge the better solution through the help of multiple parameters considered in the fitness function.

Table 4. Comparison on different protocols

\begin{tabular}{|l|l|l|l|}
\hline & $\begin{array}{l}\text { Rate } \\
(\mathrm{kb})\end{array}$ & Delay $(\mathrm{sec})$ & $\begin{array}{l}\text { Delivery } \\
\text { Ratio }\end{array}$ \\
\hline BREQRP & 250 & $\mathbf{0}$ & $\mathbf{1}$ \\
\hline IAMQER [14] & 250 & 4 & 0.8 \\
\hline AMRA [23] & 250 & 7.5 & 0.22 \\
\hline MAREERP [23] & 250 & 6 & 0.3 \\
\hline Dijkstra [14 & 250 & 8.8 & 0.95 \\
\hline IAMQER [14] & 250 & 9.5 & 0.96 \\
\hline NQoS AODV [14] & 250 & 9 & 0.98 \\
\hline
\end{tabular}

\section{Conclusion}

In this paper, we have proposed backup route establishment for QoS routing protocol. The proposed BREQRP was performed using two important phases. In the first phase, the primary routes are discovered using GA-based routing algorithm where, link quality metrics were incorporated. In the second phase, the path evaluation function is utilized to find the node failure using energy, interference and congestion. In this protocol, the potential failures of network and node are identified and a backup routing is initiated. Once failure is detected, back up routes are established and transmission is redirected on these back up routes. Simulation results show that the proposed protocol has reduced recovery delay and improved throughput. Overall, the proposed BREQRP outperforms IAMQER by $68 \%$ in terms of delay, $30 \%$ in terms of delivery ratio and $83 \%$ in terms of throughput. In future, the proposed BREQRP can be improved by adding more security features to bring more robustness among various network-related attacks.

\section{References}

[1] P.Deepalakshmi, and S. Radhakrishnan, "QoS routing algorithm for mobile ad hoc networks using ACO", in proceedings of International Conference on Control, Automation, Communication and Energy Conservation, INCACEC 2009.

[2] R.Yadav,S.Yadav,A. Yadav, and S. Bhardwaj, "QoS Multipath Path Routing in MANET", National Workshop-Cum-Conference on Recent Trends in Mathematics and Computing (RTMC),Journal of Computer Applications (IJCA), 2011.

[3] Ivascu, G.Ioan, S.Pierre, and A. Quintero. "QoS routing with traffic distribution in mobile ad hoc networks" Computer Communications, Vol. 32, No. 2, pp. 305-316, 2009.

[4] Yiltas, Derya, and H.Perros, "QoS-based multidomain routing under multiple QoS metrics", IET Communications, Vol. 5, pp 327-336, 2011.

[5] Yussof, Salman, and H.S.Ong, "A robust GAbased QoS routing algorithm for solving multiconstrained path problem" Journal of Computers, Vol. 5, No. 9, pp. 1322-1334, 2010.

[6] C.T.Calafate, M.P. Malumbres, J.Oliver, J.C.Cano and P.Manzoni, "QoS support in MANETs: a modular architecture based on the IEEE 802.11e technology", IEEE Transactions on Circuits and Systems for Video Technology,Vol. 19, No. 5,pp. 678-692, 2009.

[7] V.Thilagavathe and K. Duraiswamy, "Adhoc on Demand Multipath Reliable and Energy Aware Quality of Service Routing for Mobile Adhoc Networks", Journal of Computer Science, Vol. 8. No. 2, pp. 181, 2012.

[8] S.J Lee and M. Gerla, "AODV-BR: Backup Routing in Ad hoc network," in Proc. IEEE WCNC, Vol. 3, 2000, pp. 1311-1316, 2000.

[9] W.P Chen and J. C. Hou, "Dynamic, Ad-hoc Source Routing with Connection- Aware LinkState Exchange and Differentiation," in Proc. IEEE Globecom '02, Vol.1, pp.188-194, 2002.

[10] M. Spohn and J. J. Garcia-Luna-Aceves, "Neighborhood Aware Source Routing," in Proc. ACM MobiHoc 2001, pp. 11-21, 2001. 
[11] W.T. Chen and W.T. Lee, "Dynamic AODV Backup Routing in Dense Mobile Ad- Hoc Networks", Wireless On-Demand Network Systems, Vol. 2928, pp 158-170, 2004.

[12] F.D.Rango and M.Tropea, "Energy saving and Load Balancing in Wireless Ad Hoc Networks through Ant-based Routing", SPECTS 2009.

[13] M.Canales, J.R.Gallego, A. HernandezSolana and A.Valdovinos, "QoS provision in mobile ad hoc networks with an adaptive crosslayer architecture", Wireless Networks, Vol. 15, pp. 1165-1187, 2009.

[14] W.Ya-li, S.Mei, W.Yi-fei, W.Ying-he and W.Xiao-jun," Improved ant colony-based multiconstrained QoS energy-saving routing and throughput optimization in wireless Ad-hoc networks", The Journal of China Universities of Posts and Telecommunications, Vol. 21, No. 1, pp. 43-53, 2014.

[15] L.C. Llewellyn, K.M. Hopkinson and S. R. Graham, "Distributed Fault-Tolerant Quality of Wireless Networks", IEEE Transactions on Mobile Computing, Vol. 10, No. 2, 2011.

[16] L.Hanzo, and R.Tafazolli, "QoS-Aware Routing and Admission Control in ShadowFading Environments for Multirate MANETs", IEEE Transactions on Mobile Computing, Vol. 10, No. 5, 2011.

[17] L.Gu, Z.Niu, J.Lv and H.Yoshiuchi, "An NAK-based Hierarchical ARQ Scheme for Reliable Data Multicast in Integrated Communication and Broadcast Networks", in Proceedings of APCC, 2008.

[18] V.Chaudhary, U.Patel, Shivaji, and Rakesh Kumar, " AODV-MBR: A New Routing Protocol with Modified Backup Approach", Wireless Networks and Computational Intelligence, Vol. 292, pp 66-73, 2012.

[19] W.K.Lai, S.Y. Hsiao, Y.C. Lin, "Adaptive backup routing for ad-hoc networks", Computer Communications, Vol. 30, pp. 453-464, 2007.

[20] N.Jaisankar and R.Saravanan, "An Extended AODV Protocol for Multipath Routing in MANETs", IACSIT International Journal of Engineering and Technology, Vol.2, No.4,2010.

[21] E.Zamani and M. Soltanaghaei, "The Improved Overhearing Backup AODV Protocol in MANET", Journal of Computer Networks and Communications, Vol.2016.

[22] The Network Simulator (NS-2) from http://www.isi.edu/nsnam/ns/

[23] S.Nallusamy, S.Appavupillai and S.Ponnusamy, "Mobile Agents based Reliable and Energy Efficient Routing Protocol for MANET", International Journal of Intelligent Engineering and Systems, Vol.9, No.3, 2016. 